\title{
Potete contare sulla nostra risposta
}

Vittorio Coletti

PUBBLICATO: 10 GENNAIO 2020

\section{Quesito:}

Alcuni lettori chiedono la ragione della differenza nella reggenza preposizionale tra contare su qualcuno e contare su di me, su di te e simili. Altri invece si chiedono che tipo di complemento sia quello retto da contare (su).

\section{Potete contare sulla nostra risposta}

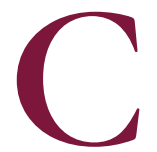

i sono due serie di domande intorno alla reggenza di contare, una di ordine linguistico e una di tipo metalinguistico.

Cominciamo da quelle della prima serie. In realtà, queste non ci interrogano sul verbo ma sulla preposizione che gli è necessaria per legarsi al complemento occorrente al suo uso intransitivo per trasmettere il significato di 'fare affidamento su qualcuno o su qualcosa', uno dei vari significati e costrutti di questo verbo decisamente polisemico. La preposizione è su e l'italiano, quando alle preposizioni improprie (sotto, dentro, dopo ecc.) o, tra le proprie, a su (che può essere anche avverbio) segue un pronome personale, preferisce che tra preposizione e pronome ci sia (ad abundantiam, cioè pleonastica) anche una seconda preposizione propria: di. Quindi "dentro l'azienda" ma "dentro di lui", "sotto il tetto" ma "sotto di me", "sul divano" ma "su di te". L'inserimento della preposizione propria di doposu e le preposizioni improprie e prima di un pronome personale ha soprattutto ragioni eufoniche (i pronomi personali complemento sono monosillabi tonici o bisillabi e il riempimento della preposizione agevola la pronuncia del sintagma) ed è, come si diceva, pleonastica, quindi opzionale o regolata caso per caso: per cui "dentro l'azienda"/ "dentro di sé", "sotto il tavolo"/ "sotto di te", "su un foglio"/ "su di voi" / "su voi".

Una parentesi. Possono essere pleonastiche e quindi opzionali anche altre preposizioni in altri sintagmi preposizionali ("dentro l'azienda"/ "dentro all'azienda", "sotto la dispensa/ sotto alla dispensa, "fuori le mura"/ "fuori dalle mura"); in certi altri sintagmi possono invece essere necessarie ("vicino a casa/ *vicino casa", "davanti alla porta/**davanti la porta").

Allora, per rispondere alla prima serie di domande: "conto su di me, su di te, su di lui", anche se "conto su Giovanni, su mio fratello, sulla buonuscita" ecc., e pure "conto su me stesso", "conta su te stesso" ecc. perché l'aggettivo dimostrativo (stesso) arricchisce il sintagma e rende meno necessaria l'inserzione eufonica della preposizione di. Ma certo mai "su di chi" (non c'è pronome personale), mentre, se è ammissibile (cfr. Serianni r989, VIII 96) "su di un servizio" (inserimento eufonico della preposizione di per interrompere la sequenza di $u$ ), è ormai più comune, preferibile e consigliabile "su un servizio" (le vecchie regole eufoniche dell'italiano stanno cambiando sotto la pressione di pronunce non più solo toscane o centroitaliane, come si sa).

E veniamo alla seconda serie di domande che chiedono lumi su quale sia il complemento retto da contare nel significato in questione di "fare affidamento su". Bisognerebbe ripetere qui per l'ennesima volta che l'ossessione classificatoria dei complementi è un vecchio e depistante residuo della nostra grammaticografia, che spesso finisce per inciampare in assurdi casi insolubili (andare a piedi è modo o mezzo?). Ma, venendo alla domanda, possiamo, per rispondere, osservare la natura locativa della 
preposizione su, soprattutto di stato in luogo. Ora, una delle valenze dello stato in luogo è quella di "poggiare" su un sostegno, come in "il vaso sta sul tavolo, il tetto poggia sulla trave". E il sostegno, si sa, può essere proprio (come quello di un tavolo o di una trave) o figurato, come quello della persona o cosa su cui si fa affidamento, che si spera o, appunto, si conta che ci sostenga ("conto sullo zio", "conto su di te", "conto su un prestito"). Come vogliamo chiamarlo questo complemento? Se proprio vogliamo dargli un nome, chiamiamolo "di (stato in) luogo", eventualmente aggiungendo, per i dubbiosi: "figurato": del resto il clitico corrispondente è ci (ci contavo 'contavo su questo'). In fondo, su chi contiamo che ci sostenga staremmo (stato in luogo) volentieri!

\section{Cita come:}

Vittorio Coletti, Potete contare sulla nostra risposta, "Italiano digitale", 2020, XII, 2020/1 (gennaiomarzo)

DOI: $10.35948 / 2532-9006 / 2020.3315$

Copyright 2020 Accademia della Crusca

Pubblicato con licenza creative commons CC BY-NC-ND 